\title{
INTERPRETIVE SUMMARIES, JUNE 2010
}

Microscopic observation of multispecies biofilm of various structures on whey concentration membranes. By Hassan et al., page 2321. Membrane separation technology has been used for decades in milk and whey processing. Biofilms are viable and nonviable single or multispecies communities of microorganisms embedded in extracellular polymeric substances. Scanning electron microscopy provided evidence of extensive biofilm formation on reverse osmosis membranes used for whey processing. Biofilm formation poses serious economic and safety concerns for the dairy industry. The extent of biofilm formation and the presence of live cells on separation membranes after regular cleanin-place cycles indicate the need for a more effective cleaning regimen customized for dairy systems.

Nucleotides and nucleosides in ovine and caprine milk during lactation. By Plakantar et al., page 2330. Nucleotides are ubiquitous intracellular compounds of crucial importance to cellular function and metabolism. They may become essential when the endogenous supply is insufficient for normal function. Ovine and caprine milk have a considerable impact on Mediterranean economy and attract a growing interest worldwide. The present study was motivated by the nutritional significance of nucleotides and the fact that little information is available regarding their presence in sheep and goat milk. We have shown that caprine and ovine colostrum and milk are rich in nucleotides.

The effect of ethanol and heat on the functional hydrophobicity of casein micelles. By Trejo and Harte, page 2338. Milk proteins are a very important ingredient to the food industry. Therefore, it is important to understand their physicochemical properties when subjected to processing operations. The changes to the functional hydrophobicity of milk proteins, when heated in the presence of ethanol, were evaluated by using the fluorescent hydrophobic probe 1-anilinonaphthalene-8-sulfonic acid. Results showed that when skim milk samples containing more than $30 \%$ ethanol were heated, the fluorescence intensity decreased because of a competitive inhibition of the probe by ethanol as well as possible changes in the protein globular structure.

Apoptosis of stomach cancer cell line SGC-7901 and regulation of Akt signaling induced by bovine lactoferrin. By Xu et al., page 2344. Bovine lactoferrin has long been thought to be a potent inhibitor of growth and transfusion to cancer. In this paper, we report bovine lactoferrin has ability to induce stomach cancer cell SGC-7901 apoptosis by 3-(4,5)-dimethylthiahiazo(z-y1)-3,5-diphenytetrazoliumromide assay and flow cytometry. Further, we show that lactoferrin inhibits Akt activation, which involves regulating growth factor- mediated cell survival by phosphorylating in both normal cell and human cancers. Thus, this study revealed a downregulation mechanism of stomach cancer growth by lactoferrin. Our findings confirm the inhibition of stomach cancer by lactoferrin as an active component in milk proteins and suggest that lactoferrin plays a potential therapeutic role in stomach cancer by inhibiting Akt activation and modulating its downstream protein phosphorylation in apoptosis. These results may also have implications in the biological treatment of human stomach cancer.

Short communication: Influence of pasteurization on the active compounds in medicinal plants to be used in dairy products. By Jäger et al., page 2351. Interest from the dairy industry in adding herbal drugs to milk products raises the question of whether these plant materials can be pasteurized. The present study investigated the effect of pasteurization on the active ingredients in 3 well-known booster plants. The results showed that ginseng and Russian root withstood the procedure, whereas Arctic root did not. These results highlight that plant materials with beneficial effects cannot always effectively be added to dairy products because the pasteurization process may destroy the effects of the plants.

Short communication: Presence of galactose and glucose promotes browning of sweet whey powder. By Dattatreya et al., page 2354. The dairy manufacturing industry produces numerous dry dairy ingredients such as nonfat dry milk and sweet whey powder. Derived from milk or cheese whey, dry dairy ingredients are compositionally complex and thus are susceptible to various reactions during storage, such as lipid oxidation or Maillard browning. Maillard browning causes undesirable changes in color and flavor character during storage, resulting in an ingredient of dramatically reduced function and economic value. The chemical basis that predisposes a dry dairy powder to develop Maillard browing is not well understood. This work demonstrates that the sugar type and $\mathrm{pH}$ play significant roles in affecting these browning reactions.

Technical note: Rapid method for determination of amino acids in milk. By Marino et al., page $236 \%$. A rapid method for amino acids (AA) measurement in milk was developed and validated. Six combinations of hydrolysis agent and temperature-time condition were compared with a reference method; derivatization procedures as well as HPLC separation were improved. Hydrolysis of milk samples with $6 \mathrm{~N} \mathrm{HCl}$ at $160^{\circ} \mathrm{C}$ for 60 min evidenced no significant differences with the reference method but allowed the analysis of an increased number of milk samples in short time. In addition, 
this method was characterized by high precision, low repeatability uncertainty, and high accuracy for all AA evaluated; the recovery mean value of the single AA was $98.38 \%$. The proposed method is, therefore, accurate, simple, rapid, and suitable for large numbers of milk samples.

Lack of association between the occurrence of Crohn's disease and occupational exposure to dairy and beef cattle herds infected with $M y$ cobacterium avium subspecies paratuberculosis. By Qual et al., page 2371. A study was conducted to identify associations between Crohn's disease (CD) and exposure to Mycobacterium avium ssp. paratuberculosis, the cause of Johne's disease. A questionaire was used to collect information. There were 3 cases of CD in 702 producers and 4 cases in 774 veterinarians, yielding a prevalence rate of $0.47 \%$. No association was found between exposure to Johne's disease and CD in any of the statistical analyses.

Prediction of ammonia emission from dairy cattle manure based on milk urea nitrogen: Relation of milk urea nitrogen to ammonia emissions. By Burgos et al., page 237\%. Lactating multiparous Holstein cows grouped by lactation stage were fed a total mixed ration containing 4 dietary crude protein levels $(15,17,19$, and $21 \%$ of diet dry matter). Urine and feces were mixed in proportions excreted by each cow to make slurries that were used to determine ammonia emissions. Ammonia emissions were not different between lactation stages but increased linearly with crude protein levels. Milk urea nitrogen concentration was linearly related to ammonia emissions and is one of several factors that allows prediction of ammonia emissions from dairy cattle manure.

Effects of encapsulated niacin on evaporative heat loss and body temperature in moderately heat-stressed lactating Holstein cows. By Zimbelman et al., page 238\%. This study evaluated the effects of feeding encapsulated niacin on skin surface temperature, evaporative heat loss, core body temperature, and production in lactating Holstein cows subjected to moderate thermal stress. Supplemented cows displayed increased evaporative heat loss and reduced core body temperature. Results support further research on the feeding of encapsulated niacin as a tool to increase insensible heat loss in lactating dairy cows.

Relationship between cortisol response to stress and behavior, immune profile, and production performance of dairy ewes. By Caroprese et al., page 2395. After an isolation test, dairy ewes were selected according to their cortisol concentration and divided into ewes with a high cortisol concentration and ewes with a low cortisol concentration to study the immune profiles, behavioral responses, and production performances connected to the different extent of activation of the hypothalamic-pituary-adrenal axis. The existence of indicators of stress-induced immune activation in milk was also studied. Results suggest that activation of the hypothalamic-pituary-adrenal axis is related to the behavioral responses and immune competence of dairy ewes and cytokine concentrations.

Effects of the peroxisome proliferator-activated receptor- $\alpha$ agonists clofibrate and fish oil on hepatic fatty acid metabolism in weaned dairy calves. By Litherland et al., page 2404. Peroxisome proliferator-activated receptor- $\alpha$ (PPAR $\alpha)$ controls fatty acid oxidation in nonruminants, and PPAR $\alpha$ activators such as clofibrate and fish oil increase fatty acid oxidation in liver. In this experiment, clofibrate and fish oil were administered to weaned dairy calves to determine responsiveness of bovine liver to compounds known to be PPAR $\alpha$ agonists in nonruminants. Clofibrate modestly increased hepatic fatty acid oxidation and expression of genes for fatty acid metabolizing enzymes, but fish oil had little effect.

Characterization of mammary stromal remodeling during the dry period. By De Vries et al., page 2433. During the dry period, the bovine mammary gland remodels in preparation for the next lactation. Changes in the epithelium during the dry period have been documented, but remodeling of the stromal tissue surrounding and supporting the epithelium has not been reported. Changes in cell and protein composition of intralobular stroma were measured throughout the dry period to gain insight into why the dry period is required to achieve maximal milk yield in dairy cows.

Border disease virus seroprevalence correlates to antibodies in bulk tank milk and reproductive performance of dairy sheep flocks. By García-Pérez et al., page 2444. Border disease virus causes abortion, stillbirth, and unviable lambs in infected pregnant ewes. Border disease virus is also able to persistently infect sheep, leading to persistently infected (PI) animals that shed the virus for life. Identifying and eliminating PI animals is the most effective control measure of border disease virus in sheep. This study shows that the serological analysis by enzyme-linked immunosorbent assay of a single bulk tank milk sample and a small number of sera from animals of different age classes is an economical first approach to evaluating the presence of PI animals in the flock.

The effect of meloxicam on behavior and pain sensitivity of dairy calves following cautery dehorning with a local anesthetic. By Heinrich et al., page 2450. An experiment was undertaken to 
investigate the effectiveness of meloxicam for relieving pain following dehorning of dairy calves, as measured by behavior. Frequency of ear-flicking and head-shaking as well as total activity, pressure algometry sensitivity, and feed intake were used as indicators of pain and were measured at regular intervals following a sham treatment and cautery dehorning. Meloxicam-treated calves displayed less pain-related behaviors, were less active following dehorning than controls, and were less sensitive to pressure algometry.

Factors associated with concentrations of select cytokine and acute phase protein in dairy cows with naturally occurring clinical mastitis. $B y$ Wenz et al., page 2458. An observational study was performed to better understand factors associated with the innate immune response of dairy cattle during naturally occurring clinical mastitis. Factors evaluated included systemic disease severity, milk culture result, parity, length of lactation, previous clinical mastitis occurrence, and season of the year. This study provided the most comprehensive report to date of the concentrations of innate immune response proteins in milk from the affected mammary gland of cows with clinical mastitis. It also identified factors that may influence those concentrations, particularly systemic disease severity and season. These results provided data useful for the design and interpretation of future studies of the innate immune response to clinical mastitis in dairy cattle.

Effects of continuous milking during the dry period or once daily milking in the first 4 weeks of lactation on metabolism and productivity of dairy cows. By Schlamberger et al., page 2471. The study investigated the effect of continuous milking throughout the entire previous lactation and milking only once daily in early lactation on metabolic profile and productivity of dairy cows. Both treatments may improve the metabolic imbalance in early lactation, whereas lactation energy-corrected milk yield (305 d) was reduced by $16 \%$. Milk protein content was increased by 0.3 to $0.5 \%$ for both management tools compared to control cows.

Assessment of the productivity effects associated with epizootic hemorrhagic disease in dairy herds. By Kedmi et al., page 2486. Epizootic hemorrhagic disease virus is an emerging virus that affects cattle. Because of the risk that new types of epizootic hemorrhagic disease will invade Europe and the United States, its economic impact needs to be estimated. We analyzed milk loss and mortality during an outbreak that occurred in Israel in 2006. Average milk loss of 204 $\mathrm{kg}$ of milk/cow and mortality of $1.42 \%$ were estimated for severely affected farms and $84 \mathrm{~kg}$ of milk/cow for mildly affected farms. Estimated production losses to the Israeli dairy industry because of milk loss and mortality were $\$ 2.491$ million, $81 \%$ of which was attributed to milk loss.

Sex-sorted semen for dairy heifers: Effects on reproductive and lactational performances. By Chebel et al., page 2496. Use of sex-sorted semen has been recommended for heifers because they generally have better pregnancy per artificial insemination $(\mathrm{P} /$ $\mathrm{AI})$, thus moderating the effect of the reduction in $\mathrm{P} /$ AI generally observed with sex-sorted semen. Heifers inseminated with sex-sorted semen had P/AI 11.5\% units smaller than heifers inseminated with conventional semen and, although sperm sorting was $85.7 \%$ accurate, insemination with sex-sorted semen resulted in only $79.1 \%$ of live female calves because heifers pregnant to sex-sorted semen were more likely to deliver dead calves. Because of longer interval from first AI to calving, heifers inseminated with sex-sorted semen had greater rearing cost, but because they delivered more female calves their economic return during the first lactation was improved.

Lipomobilization in periparturient dairy cows influences the composition of plasma nonesterified fatty acids and leukocyte phospholipid fatty acids. By Contreras et al., page 2508. The mobilization of lipids from tissue stores occurs during the periparturient period when dairy cattle experience increased energy requirements. The objective of this study was to characterize the fatty acid profiles of plasma lipid fractions and determine the fatty acid content of peripheral blood mononuclear cells obtained from periparturient cows. Results demonstrated that palmitic and stearic acids are the main components of plasma NEFA and phospholipids of leukocytes during lipomobilization, whereas concentrations of long-chain polyunsaturated fatty acids in both fractions were reduced. Increased availability of saturated fatty acids and reduced sources of polyunsaturated fatty acids could predispose leukocytes to enhanced proinflammatory activity.

Assessing pest control using changes in instantaneous rate of population increase: Treated targets and stable fly populations case study. By Beresford and Sutcliffe, page 251\%. In temperate climates, stable fly populations grow over the summer until being killed by the arrival of cold winter temperatures. In 2001, attractive targets treated with pesticide were tested to assess reduction in stable fly population growth rates on dairy farms in Ontario. Over a 10-wk period, 3 farms were outfitted with attractive targets treated with permethrin and 3 had untreated targets. Stable fly populations grew significantly slower at farms with treated permethrin. A long-term management 
strategy that slows population growth rates should be adopted to delay when stable flies become economically significant pest.

Ovsynch versus Ultrasynch: Reproductive efficacy of a dairy cattle synchronization protocol incorporating corpus luteum function. $B y$ McArt et al., page 2525. Reproductive efficiency is an important aspect of profitability of dairy farms, and synchronization protocols have been developed to increase pregnancy rate and decrease reliance on estrus detection. This study compared the reproductive efficiency of Ultrasynch, a synchronization protocol based on functionality of the corpus luteum, with a standard Ovsynch protocol for cows found open at pregnancy examination. There was no difference in median days to conception between the 2 treatments.

Estrone and $17 \beta$-estradiol concentrations in pasteurized-homogenized milk and commercial dairy products. By Pape-Zambito et al., page 2533. Estrone and $17 \beta$-estradiol were quantified in commercial skim, $1 \%, 2 \%$, and whole milk samples and half-andhalf, cream, and butter samples from products with organic and conventional labels. $17 \beta$-Estradiol also was quantified in milk pre- and post-pasteurization-homogenization. $17 \beta$-Estradiol concentrations in milk were not different pre- and post-pasteurization-homogenization. Estrone and $17 \beta$-estradiol concentrations in milk samples were correlated significantly with fat content in milk and concentrations did not differ between conventional and organic milk samples. The quantities of free estrone and $17 \beta$-estradiol that would be consumed in commercially available dairy products are low relative to endogenous production rates in humans.

Effect of automatic cluster remover settings on production, udder health, and milking duration. By Jago et al., page 2541. The effects of 4 different automatic cluster remover settings on milking duration, milk production, teat condition, and mastitis were examined. Using a milk flow rate threshold setting of $0.4 \mathrm{~kg} / \mathrm{min}$ or $0.2 \mathrm{~kg} / \mathrm{min}$, combined with a maximum cluster attachment time, applied from either early or peak lactation, reduced milking duration compared with a control setting of $0.2 \mathrm{~kg} / \mathrm{min}$. Over a period of $35 \mathrm{wk}$ no differences in milk production, teat condition, or clinical mastitis were detected. The somatic cell count was low for all treatments. Reducing cluster attachment duration can reduce milking time without affecting milk production, clinical mastitis, or teat condition and is unlikely to result in an increase in somatic cell count.

Host adaptation of bovine Staphylococcus aureus seems associated with bacteriological cure after lactational antimicrobial treatment. By van den Borne et al., page 2550. Multilocus sequence types of bovine subclinical Staphylococcus aureus mastitis isolates was determined to investigate their association with bacteriological cure after antimicrobial treatment during lactation. Sequence types identified in multiple host species had a better probability of bacteriological cure than sequence types observed primarily in bovine milk samples. Penicillin resistance was more common in nonbovine-associated strains than in bovine-associated strains. Genotyping of Staphylococcus aureus might have diagnostic value in dairy practice when subclinical mastitis treatment decisions have to be made.

Short communication: Repeatability of differential goat bulk milk culture and associations with somatic cell count, total bacterial count, and standard plate count. By Koop et al., page 2569. Culturing bulk milk can be used as a tool to identify possible causes of high standard plate count or somatic cell count. In this study, we show that the staphylococcal count is related to the somatic cell count and to the total bacterial count in dairy goat bulk milk. This information may be useful for goat farms with somatic cell count or total bacterial count problems.

Technical note: A rapid method for 5-bromo2'-deoxyuridine (BrdU) immunostaining in bovine mammary cryosections that retains RNA quality. By Choudhary et al., page 2574. 5-Bromo-2'deoxyuridine (BrdU) is a synthetic nucleotide that is incorporated into the cell's DNA when it is proliferating. Coupled with antibody-based histological methods, BrdU is a powerful agent for identifying proliferating cells. Because methods for detecting cells that have incorporated BrdU are harsh, it has not been feasible to obtain good-quality RNA from these tissue sections. A protocol was developed for detecting BrdU-labeled cells in histological sections, which does not destroy RNA. This permits recovery of RNA specifically from isolated, BrdU-labeled cells to study gene regulation of cell proliferation.

Flaxseed supplementation improves fatty acid profile of cow milk. By Caroprese et al., page 2580. Two sources of fatty acid supplementation, flaxseed and fish oil, were added to dairy cow diet in order to evaluate the effects on milk composition and fatty acid profile during summer. Flaxseed supplementation enhanced fatty acid profile of cow milk in terms of conjugated linoleic acid isomers content. Milk from cows supplemented with flaxseed was improved in its nutritional and health features, suggesting that its consumption is beneficial for health.

Effect of dietary modifications of calcium and magnesium on reducing solubility of phosphorus in feces from lactating dairy cows. By Her- 
rera et al., page 2598. Recommended concentrations of dietary phosphorus have been reduced in recent years to minimize movement of phosphorus from dairy farms. Solubility of phosphorus from dairy cow feces was reduced preemptively by increasing dietary concentration of calcium from an average of 0.64 to $0.95 \%$ when cows were fed the recommended dietary concentration of phosphorus. This can potentially reduce the involuntary off-farm movement of phosphorus. Feeding more magnesium also was effective but not to the same extent as calcium. Calcium carbonate is a preferred calcium source to calcium chloride.

Supplementation with whole cottonseed causes long-term reduction of methane emissions from lactating dairy cows offered a forage and cereal grain diet. By Grainger et al., page 2612. We examined the effects of supplementing a forage and cereal grain diet with whole cottonseed over a 12 -wk period on enteric methane emissions and milk yield of dairy cows. Milk yield was reduced with whole cottonseed supplementation, but this was attributable to a reduced total intake of the cottonseed diet resulting from the combination of hot weather conditions during the experiment and an increase in fiber content of the cottonseed diet. The addition of whole cottonseed resulted in a persistent reduction in methane emissions over a 12 -wk period, and the reduction in methane is consistent with previous work that has studied the addition of oilseeds to ruminant diets.

The relationship of silage temperature with the microbiological status of the face of corn silage bunkers. By Borreani and Tabacco, page 2620. A method is proposed to quantify the aerobic deterioration of corn silage in the bunker as related to the temperature of the ensiled silage mass. This method shows how to link aerobic microbial stability to temperature measurements at the face of the silage bunker, thus helping alert farmers to the onset of aerobic deterioration and indicating the areas with invisible altered silage masses that should be discarded and not fed to animals.

Mitigation of enteric methane emissions through improving efficiency of energy utilization and productivity in lactating dairy cows. By Yan et al., page 2630. Methane emission from dairy cows is a considerable source of greenhouse gas emissions. The present study demonstrates that increasing energy intake or milk production per cow significantly reduced methane emission as a proportion of energy intake or milk yield. Methane emission rates are negatively related to the efficiency of energy utilization for lactation and energy expenditure for maintenance in dairy cows. The selection of dairy cows with high energetic efficiencies and milk productivity offers an effective approach to reduce methane emissions from milk production.
Long-term effects of ad libitum whole milk prior to weaning and prepubertal protein supplementation on skeletal growth rate and first-lactation milk production. By Moallem et al., page 2639. Heifer calves were fed either ad libitum milk replacer or whole milk from $4 \mathrm{~d}$ of age until weaning at $60 \mathrm{~d}$ of age. From 150 to $320 \mathrm{~d}$ of age the original groups were divided into subgroups, one of which was supplemented with $2 \%$ added protein. Body weight at weaning was higher in the milk-fed calves, with no effects of nursery treatments or added protein on later growth rates. The milk-fed heifers produced $10.2 \%$ more milk during the first lactation.

Effect of water addition to a total mixed ration on feed temperature, feed intake, sorting behavior, and milk production of dairy cows. By Felton and DeVries, page 2651. The objective of this study was to determine the effects of water addition to a high-moisture total mixed ration on feed temperature, feed intake, feed sorting behavior, and milk production of dairy cows. The treatment diets averaged $56.3 \%$, $50.8 \%$, and $44.1 \%$ dry matter. The results suggest that the addition of water to high-moisture total mixed rations (less than $60 \%$ dry matter) containing primarily haylage and silage forage sources will not always discourage cows from sorting but rather may increase this behavior, limit the nutrient consumption of cows, and cause greater increases in feed temperature in the bunk, particularly when ambient temperature is high.

Saccharomyces cerevisiae fermentation product in dairy cow diets containing dried distillers grains plus solubles. By Hippen et al., page 2661. It was hypothesized that feeding Saccharomyces cerevisiae fermentation product in diets with distillers grains plus solubles would improve lactation performance and minimize the effects of distiller grains on milk fat depression in diets with minimal forage fiber. Cows fed diets containing $19.3 \%$ forage fiber and $20 \%$ distillers grains plus solubles experienced a decrease in milk fat production that was not prevented by feeding Saccharomyces cerevisiae fermentation product. However, cows fed Saccharomyces cerevisiae fermentation product did produce greater quantities of milk and milk protein.

Effect of dietary nitrogen content and intravenous urea infusion on ruminal and portal-drained visceral extraction of arterial urea in lactating Holstein cows. By Kristensen et al., page 2670. This study addressed the fundamental properties of urea transport across gut epithelia that are important for assessing the perspectives of increasing nitrogen utilization by urea recycling in cattle. The study showed that the proportion of arterial urea extracted upon passage of gut epithelia increased when cows were fed low levels of dietary nitrogen and that the dietary-induced 
changes in epithelial urea extraction were maintained when the arterial urea concentration was increased by short-term intravenous infusion of urea. Data are commensurable with a model for urea transport across gut epithelia based on regulated expression or activity of facilitative urea transporters.

Short communication: Fat-soluble vitamin and mineral status of milk replacer-fed dairy calves: Effect of growth rate during the preruminant period. By Nonnecke et al., page 2684. Growth rate during the preruminant phase of development influences not only body composition and health of the milk replacer-fed calf but may also enhances productivity later in life. The present study evaluated the effects of 3 targeted rates of gain [no-growth $(\mathrm{NG}), 0.11 \mathrm{~kg} / \mathrm{d}$; low-growth (LG), $0.58 \mathrm{~kg} / \mathrm{d}$; high-growth (HG), 1.16 $\mathrm{kg} / \mathrm{d}]$ on serum concentrations of fat-soluble vitamins and minerals in milk replacer-fed calves over a 7 -wk period. Of the vitamins examined, only the availability of vitamin $\mathrm{E}$, an essential antioxidant, appeared to be affected by growth rate. Vitamin $\mathrm{E}$ concentrations in HG calves were markedly lower than concentrations in LG and NG calves, suggesting that the level of vitamin $\mathrm{E}$ in diets of calves on nutritional programs promoting high growth rates needs to be reevaluated. Copper, calcium, and phosphorus concentrations in the blood of $\mathrm{HG}$ calves exceeded concentrations in $\mathrm{LG}$ and $\mathrm{NG}$ calves during latter weeks of the study, likely because of increased consumption of milk replacer by HG calves. Vitamin and mineral concentrations for calves in all treatment groups remained within normal ranges for young dairy calves.

Response to alternative genetic-economic indices for Holsteins across 2 generations. By Norman et al., page 2695. Genetic-economic indices for improving US dairy cattle have been available since 1971 and have become more comprehensive over time by including more traits. Various genetic-economic indices were retrofitted to demonstrate response to selection for several traits across 2 generations. The 2006 net merit index was shown to provide improvement for all traits included, and use of a comprehensive composite index should be a valuable tool for producing a dairy population that performs efficiently for many health and fitness traits.

Testing candidate gene effects on milk production traits in dairy cattle under various parameterizations and modes of inheritance. By Suchocki et al., page 2703. The study concerns the estimation of effects of single nucleotide polymorphisms (SNP) on dairy production traits. An analysis of simulated data showed that in the presence of epistasis for a fully parameterized model with additive, dominance, and epistatic SNP effects, correct confidence intervals for
SNP in epistatic relationship are provided by only a particular parameterization. When analyzing real data sets from Jersey and Holstein-Friesian breeds the additive effects of SNP located within butyrophilin, diacylglycerol acyltransferase 1, leptin, and leptin receptor candidate genes were similar between breeds, except for leptin receptor, which had different alleles that increased fat content in Holstein-Friesian and Jersey.

Random regression analysis of milk yield and milk composition in the first and second lactations of Murciano-Granadina goats. By MenéndezBuxadera et al., page 2718. This work explores the suitability of test-day records to estimate breeding values and genetic parameters of Spanish Murciano-Granadina goats, using random regression models. The same traits in first and second lactation were correlated positively, but the correlations were much less than unity; these traits are not the same genetically, as assumed by the currently used repeatability models. Important genetic variability for the shape of the lactation curve was also identified. Models presented in this work will contribute to improving the reliability of the estimations of breeding values and, therefore, to increasing the selection response in this breed.

Visualization of results from genomic evaluations. By Cole and VanRaden, page 2727. Genomic predictions of estimated breeding values include effects of tens of thousands of markers distributed over 30 chromosomes for many traits. With so many numbers, comparisons are difficult and tables quickly grow very large. Guidelines are provided for presenting genomic data graphically. Genetic variances can be plotted to compare chromosomes. Potential mates may be compared using chromosomal estimated breeding values. Interesting associations among traits can be identified using correlation matrices. Marker effects can be used to locate causative loci for new recessives. Graphics can be produced automatically and added to online query systems. High-quality graphics can improve understanding of the data.

A comparison between Holstein-Friesian and Jersey dairy cows and their F1 cross with regard to milk yield, somatic cell score, mastitis, and milking characteristics under grazing conditions. By Prendiville et al., page 2741. This study provides insight into the comparative milk yield, udder health, and milking characteristics of Holstein-Friesian, Jersey, and Jersey $\times$ Holstein-Friesian cows under grazing conditions. Although large differences in milk yield were apparent, no difference in udder health (somatic cell score or incidence of individual cows getting mastitis) and milking duration were observed between the breed groups. Average milk flow was greater with the Holstein-Friesians, with peak milk flow also tend- 
ing to be greater with the Holstein-Friesians compared with the Jerseys. Evidence also suggests that Jersey $x$ Holstein-Friesian cows may exhibit heterosis for average milk flow and peak milk flow.

Genetic analysis of fertility related diseases and disorders in Norwegian Red cows. By Heringstad, page 2751. Records of silent heat (SH), cystic ovaries (CO), metritis (MET), and retained placenta (RP) for 503,683 first-lactation daughters of 1,058 Norwegian Red sires were analyzed using a multivariate threshold model. Point estimates of heritability of liability were 0.06 for $\mathrm{SH}, 0.03$ for MET, 0.07 for $\mathrm{CO}$, and 0.06 for $\mathrm{RP}$. The genetic correlations among traits were near zero, except between MET and RP (0.64) and between RP and CO (-0.26). Although the frequency of fertility-related disorders is very low in the Norwegian Red population at present, reasonably precise genetic evaluation of sires can be obtained from large daughter groups.

Predicting energy balance for dairy cows using high-density single nucleotide polymorphism information. By Verbyla et al., page 275\%. The declining performance of nonproduction traits such as fertility and health has become a major concern to the dairy industry. Energy balance, defined as the difference between energy intake and energy usage, could be used to find a balance between production and nonproduction traits because both rely on a common source of energy. The objective of the study was to examine the genetic basis of energy balance and show that it could be incorporated into selection programs using genomic selection. Overall, the study demonstrated that genomic selection could be used to improve energy balance.

Using eigenvalues as variance priors in the prediction of genomic breeding values by principal component analysis. By Macciotta et al., page 2765. Principal component analysis with the use of eigenvalues as variance priors was effective in reducing the number of predictors up to $96 \%$ and saving computational resources for the prediction of individual genetic merit for a genome of 6 chromosomes and 6,000 single nucleotide polymorphism markers available. The same accuracy (0.76) was obtained when 279 principal components were used as predictors instead of 5,925 single nucleotide polymorphism markers. Moreover, one of the top principal components was able to depict the variation between individuals of different generations

Short communication: Characterization of the genome-wide linkage disequilibrium in 2 divergent selection lines of dairy cows. By Banos and Coffey, page 2775. With new DNA technology, it was possible to genotype 299 Holstein cows for 54,001 ge- netic markers (single nucleotide polymorphisms; SNP). Cows belonged to 2 divergent genetic lines (166 selected for fat and protein yield vs. 133 controls) raised on an experimental farm. Linkage disequilibrium was determined among SNP pairs located within 1,000,000 base intervals, separate in each line. Significantly higher linkage disequilibrium was calculated in the select line, possibly as a result of ongoing selection. The implication is that a larger number of SNP than currently available is needed for genomic evaluation and selection across divergent genetic lines.

Recall costs balanced against spoilage control in Dutch custard. By Velthuis et al., page 2779. The moment at which a recall of Dutch custard is initiated and its corresponding costs were investigated using a model to simulate the distribution of $24,000 \mathrm{~L}$ of custard over the chain over time, the time to detect Pseudomonas spp., and the direct recall costs. Recall costs increase from $€ 25,000$ to $€ 36,171$ from 57 to $135 \mathrm{~h}$ in the situation without quarantine and from $€ 25,000$ to $€ 36,648$ from $123 \mathrm{~h}$ to $163 \mathrm{~h}$ for the situation with quarantine; thereafter, the costs decrease. With low true contamination probabilities quarantine is not profitable, but at later detection moments with high probabilities it is profitable.

Biofilm in milking equipment on a dairy farm as a potential source of bulk tank milk contamination with Listeria monocytogenes. By Latorre et al., page 2792. We assessed the presence of biofilms in milking equipment as a potential source of Listeria monocytogenes contamination in bulk tank milk on a dairy farm. We observed numerous isolated bacteria and bacteria in a biofilm matrix on milk meter surfaces used in the study farm. Our results are suggestive of a Listeria monocytogenes-containing biofilm. Hygiene during milking, adequate cleaning, and regular replacement of plastic and rubber materials in milking equipment would help dairy producers to prevent biofilms and subsequent milk contamination.

Evaluation of protein fractionation and ruminal and intestinal digestibility of corn milling coproducts. By Kelzer et al., page 2803. Seven different corn milling co-products were analyzed to compare differences in chemical composition, protein fractions, protein degradation, amino acid availability, and microbial fermentation characteristics. This research demonstrates large variation in chemical composition, protein digestibility, and amino acid concentration. In addition, the proportion of protein bypassing rumen degradation is different among the different types of corn milling co-products. Measured values for chemical composition and nutrient availability are more valuable than book values when formulating livestock rations. 
Dairy Cattle Breeding Simulation Program: A simulation program to teach animal breeding principles and practices. By Medrano et al., page 2816. A dairy cattle breeding simulation program and a Web page interface have been developed to teach undergraduate and graduate students animal breeding principles associated with selection for multiple traits in dairy cattle. This software simulates a population of dairy herds and artificial insemination bulls through several generations by integrating students' decisions about mating, culling, and selection of new heifers and bulls on the basis of a multivariate animal mixed model evaluation and marker-assisted selection. After several running periods, the genetic trend can be evaluated, providing realistic experience for development of animal breeding skills relevant to students with basic animal breeding knowledge. 